MPIKG Public Access

Author Manuscript

Published in final edited form as:

Eickelmann, S., \& Riegler, H. (2018). Rupture of ultrathin solution films on planar solid substrates induced by solute crystallization. Journal of Colloid and Interface Science, 528, 63-69. doi:10.1016/j.jcis.2018.05.034.

\title{
Rupture of ultrathin solution films on planar solid substrates induced by solute crystallization
}

Stephan Eickelmann, Hans Riegler

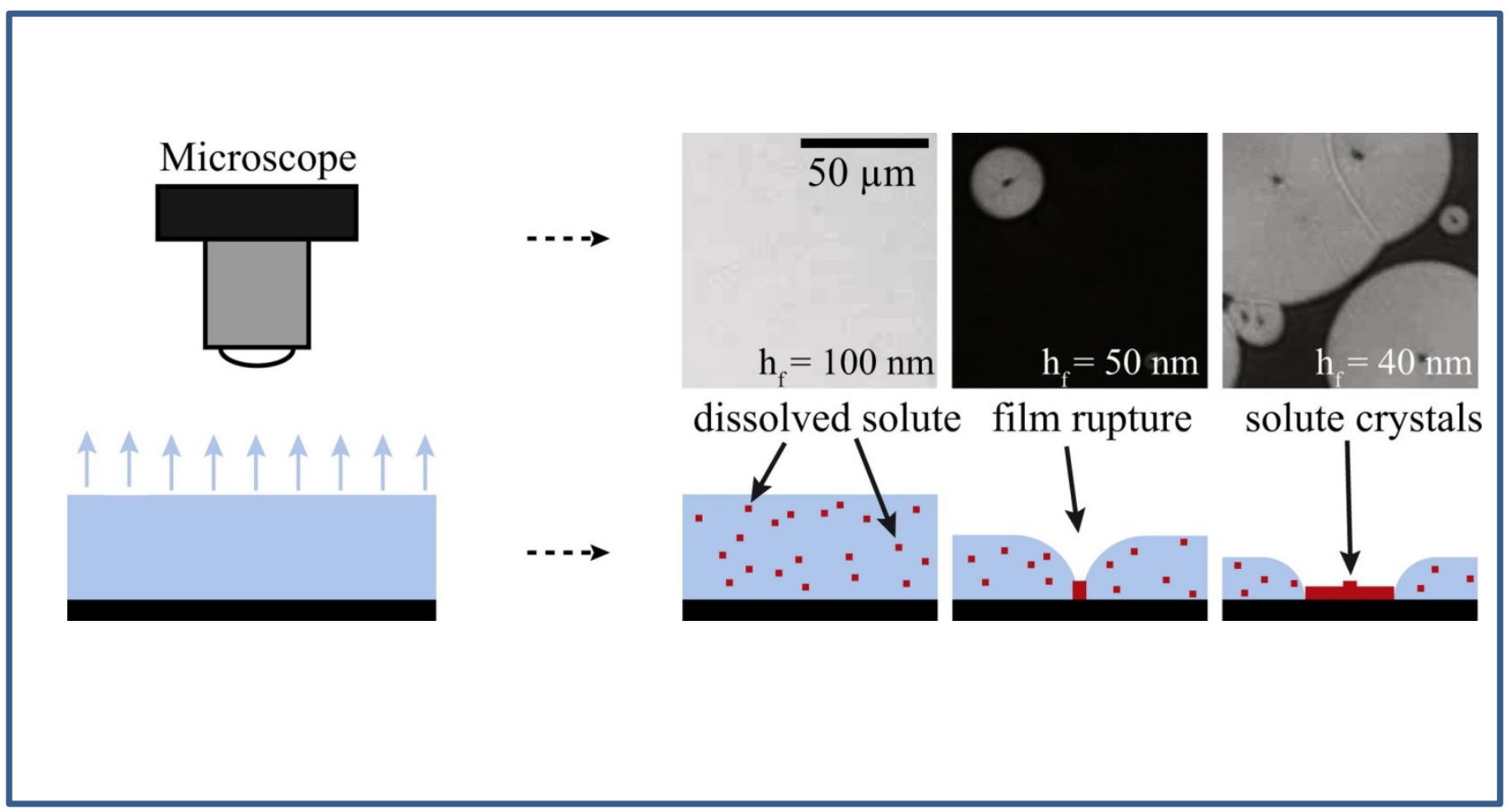

\section{Keywords}

Thin films, Film rupture, Wetting, Dewetting, Binary solutions, Solute crystallization 


\title{
Rupture of ultrathin solution films on planar solid substrates induced by solute crystallization
}

\author{
Stephan Eickelmann, Hans Riegler* \\ Max Planck Institut für Kolloid und Grenzflächen, Abteilung für Theorie und Bio-Systeme, Am Mühlenberg 1, D-14476 Potsdam, Germany
}

\begin{abstract}
On-line optical imaging of continuously thinning planar films in a spin cast configuration reveals the rupture behavior of ultra-thin films of binary mixtures of a volatile solvent and a nonvolatile solute. The pure solvents completely wet the silica substrates whereas the solution films rupture at certain film thicknesses, $h_{\text {rupture }}$, which depend on, $c_{0}$, the initial weighing in solute concentrations. With small $c_{0}, h_{\text {rupture }}$ increases proportional to $c_{0}$. With high $c_{0}$, all films rupture at $h_{\text {rupture }} \approx 50 \mathrm{~nm}$, independent of $c_{0}$. The findings can be explained by the solute enrichment during the evaporative thinning. Solute crystallization at the liquid/substrate interface upon reaching solute supersaturation leads to locally different wetting properties. This induces locally the rupture of the film as soon as it is sufficiently thin. A proper data rescaling based on this scenario yields a universal rupture behavior of various different solvent/solute mixtures.
\end{abstract}

Keywords: thin films, film rupture, wetting, dewetting, binary solutions, solute crystallization

\section{Introduction}

A liquid deposited on a solid surface may wet the surface completely or only partially. As a result it may form a planar closed film or rupture and form a film with holes, or even rearrange into individual droplets. The wetting and rupture behavior of thin films on planar solid substrates is a permanent research topic[1-5] not the least because it is of eminent practical relevance [6-11].

For liquid films thinner than millimeters gravitational forces can be neglected. Their wetting behavior is determined by the interactions between the substrate and the film [12-15]. With smooth substrate surfaces the interactions depend on the composition and state of the film (e.g. its temperature). It depends also on the film thickness and of the bulk properties of the substrate (e.g. with $\mathrm{VdW}$ interactions the disjoining pressure and its sign).

Typical wetting studies address the relation between all these parameters and the wetting and film rupture behavior. There have been experimental studies on the rupture of liquid films [16-20], as well as thin polymer or thin metal films[21-26]. The film rupture has also been investigated theoretically[2730]. In most cases investigated up to now the composition of the liquid film was kept fixed.

However, in most naturally occurring and industrially applied cases, in particular with volatile liquid components, the composition of the liquid changes with time and film thickness[31]. Aside from the film thickness this constitutional

\footnotetext{
${ }^{*}$ Corresponding author

Email addresses: stephan.eickelmann@mpikg.mpg.de (Stephan Eickelmann), hans .riegler@mpikg.mpg.de (Hans Riegler)
}

change can have a very strong impact on the interactions determining the wetting and rupture behavior. The change in film composition, for instance, may switch its behaviour from inherently wetting to non-wetting and thus lead to film rupture.

For experimental investigations addressing the impact of the (changing) film composition on the rupture behaviour the composition has to be (continuously) controlled and known while the wetting and rupture behavior is monitored. In the following we will present studies on the wetting/rupture behavior of continuously thinning liquid planar films consisting of binary solvent/solute mixtures deposited on smooth planar substrates. The wetting/rupture properties are investigated by continuously imaging a small area of the liquid film with reflection optical microscopy, while the film is continuously thinning due to the evaporation of the volatile liquid component. At the same time the nonvolatile solute gets continuously enriched in the thinning film. Practically the experiments are performed in a spin cast configuration, where the thinning of the film and the evolution of its composition is well known [32,33]. The investigations show that the film composition affects the wetting and rupture behavior. The local crystallization of the solute at the interface leads the formation of holes at these locations if the film is thin enough so that film rupture is energetically favorable.

\section{Methods and Material}

\subsection{Experimental Setup}

The wetting/rupture of the thinning liquid films is investigated in a "spin cast configuration" by time resolved reflection microscopy [34-38] as depicted in Figure 1. The microscope setup was implemented with a Axio Scope.A1 Vario from Zeiss. The images were recorded with a fast CMOS camera 


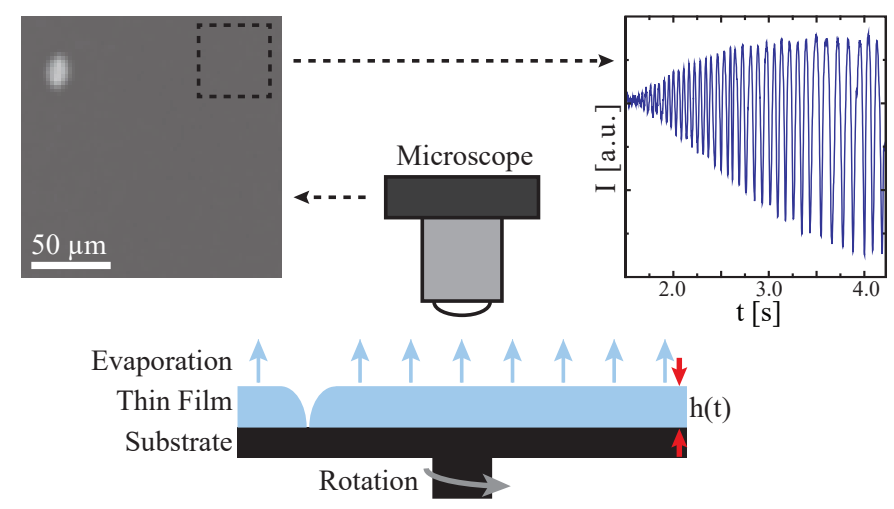

Figure 1: Experimental setup to investigate the thinning and rupture behavior of thin planar films of volatile liquids on rotating planar solid substrates. With volatile liquids and sufficiently thin films, film thinning is dominated by evaporation. A small area of the evaporating film is continuously monitored with time-resolved optical reflection microscopy. Interference effects of the various parallel planar interfaces of the system cause oscillatory brightness variations. These are used to measure the overall film thinning behavior. In addition optical imaging reveals the evolution of local structures such as holes (film rupture).

(EoSense $\AA C L$ MC1362, Mikrotron GmbH). A blue diode laser (6 W, $445 \mathrm{~nm}$, LDM-445-6000, LASERTACK) was used for illumination. The laser light was de-speckled by a combination of a liquid light guide and a rotational diffuser. The samples were placed on the sample plate of a spin coater within a homebuild environmental chamber to control/adjust the gaseous environment. The spin coater itself was mounted on a X-Y table to adjust the sample position relative to the optical microscope. An optical encoder on the spin coater rotation axis provided a periodic trigger signal for image recording at fixed rotational angles. Thus 60 images per rotation could be recorded phaselocked with the axis rotation. The images were processed and analyzed by a custom-made image analysis software. In post processing the frames were rotated and shifted relative to each other to yield a sequence (movie) of non-rotating images.

\subsection{Hydrodynamic-evaporative film thinning}

In a spin cast configuration film thinning as well as the evolution of the solvent composition are meanwhile quantitatively well understood for mixtures of non-volatile solutes and volatile solvents [32, 33]. All thinning films were prepared and investigated under constant roation speed. A small amount of liquid $(\approx 200 \mu \mathrm{l}=$ "excess volume", see [32, 33]) is deposited on the rotating planar substrate. Right after liquid deposition the interplay of hydrodynamics and viscous forces planarizes the liquid into a flat film [39]. With volatile liquids this planar film is continuously thinning due to hydrodynamically driven flow as well as evaporation [40]. Whereas at the beginning, with relatively thick films, the film thinning is dominated by hydrodynamics, at later stages its thinning is dominated by evaporation. The crossover between hydrodynamic and evaporative film thinning occurs at the so-called transition height $h_{t r}[32,33]$ :

$$
h_{t r}=(E / 2 K)^{1 / 3}
$$

( $K=$ "Spin-Off" coefficient, $\omega=$ rotational speed, and $v=$ kinematic viscosity) With typical evaporation rates of $E=$
$1.5 \mu \mathrm{m} / \mathrm{s}$ and typical spin cast parameters of $K=\omega^{2} /(3 v)=$ $5 \cdot 10^{-9} \mathrm{~mm}^{-2} \mathrm{~s}^{-1}\left(\omega=1000 \mathrm{rpm}, v=0.65 \mathrm{~mm}^{2} / \mathrm{s}\right) h_{t r}$ is in the range of a few $\mu \mathrm{m}$. Therefore, as soon as the films are thinner than $\approx \mu \mathrm{m}$ the time evolution of the film thickness is dominated by evaporation.

\subsection{Film thickness determination and thickness/time resolution}

The system of the thinning film on the solid substrate contains several parallel planar interfaces. Under illumination in the reflection microscopy configuration these interfaces lead to optical interference effects. As a result the reflected intensity varies during the film thinning. These intensity variations are used to determine the liquid film thickness. With the addition of interference enhancement through artificially grown $\mathrm{SiO}_{2}$ layers on the substrates and image processing (background subtraction, oversampling) the liquid film thickness can be determined with nm resolution $[41,42]$ (the lateral imaging resolution is diffraction-limited and in the range of about $500 \mathrm{~nm}$ ). With the installed camera (typical frame rate: $1000 \mathrm{~s}^{-1}$, exposure time for each individual frame $<80 \mu$ s) individual images can be recorded for film thickness increments of typically nanometers.

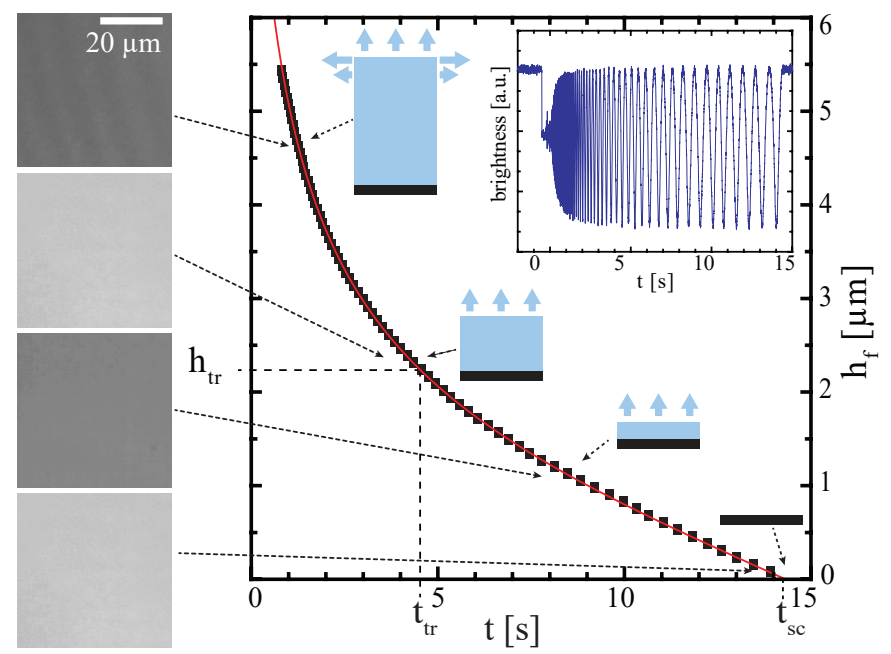

Figure 2: Time resolved imaging data and corresponding time dependent film thickness curve measured from the hydrodynamic-evaporative thinning of toluene on a rotating $\mathrm{SiO}_{2}$ substrate $(\omega=1000 \mathrm{rpm})$. The transition height $h_{t r}$ characterizes the transition between the early (hydrodynamic) stage of film thinning controlled by viscous forces, and the later stage determined by liquid evaporation. Experiments for this report focus on the very late stages of film thinning with film heights, $h_{f}$, smaller than $100 \mathrm{~nm}$. In this case $\left(h_{f}<<h_{t r}\right)$ film thinning is dominated by evaporation.

\subsection{Example of a film thinning measurent}

Figure 2 shows an example of the hydrodynamic-evaporative thinning of a film of pure toluene performed in a spin cast configuration. The imaging of the planar film shows uniform gray areas with oscillating gray levels as the film is thinning. The combination of gray level variation, (known) refractive indices and (known) illumination wavelength yields the film thinning curve [43]. It is characteristic for the hydrodynamicevaporative thinning of a film of a volatile liquid in a spin 
cast configuration $[32,33]$. It starts with a rapid, non-linear decrease of the film thickness driven by hydrodynamics. Then the thinning slows down and eventually it proceeds linearly until complete drying. During this linear thickness decrease film thinning is dominated by evaporation. Essentially it is a planar liquid film on a planar solid substrate, which is evaporating with a constant evaporation rate $E$. In particular in the late stages of the film thinning hydrodynamic effects, such as lateral shear flow can be neglected.

\subsection{Materials}

Solvents: Toluene $\left(\mathrm{C}_{7} \mathrm{H}_{8}\right.$, Chromasolv Plus for HPLC, $99.9+\%)$, methylcyclohexane $\left(\mathrm{MCH}, \mathrm{C}_{7} \mathrm{H}_{14}\right.$, high purity $99.5 \%$, Sigma-Aldrich), n-nonane $\left(\mathrm{C}_{9} \mathrm{H}_{20}\right.$, high purity $99.8 \%$, Sigma-Aldrich), and deionized water (MilliQ).

Solutes: n-Hexatriacontane $\left(\mathrm{C}_{36} \mathrm{H}_{74}\right.$, high purity $99.99 \%$, Sigma-Aldrich), n-triacontane $\left(\mathrm{C}_{30} \mathrm{H}_{62}\right.$, high purity $99.99 \%$, Sigma-Aldrich), and $\mathrm{NaHCO}_{3}(99.7+\%)$.

Substrates: Single sided, polished silicon wafers (( $\mathrm{Si}(100)$, p-dotated, 1 - $30 \Omega \mathrm{cm}$, Siegert Wafer, Aachen, Germany) with an artificially grown oxide layer (wet oxidation) of 50 $\mathrm{nm}$ thickness. The wafers were cut into substrates of roughly $2 \times 2 \mathrm{~cm}$.

Substrate Cleaning: The substrates were sonicated in a ultrasonication bath for $5 \mathrm{~min}$ each in a sequence of MilliQ, ethanol, acetone, ethanol, and MilliQ-water. Then they were cleaned with piranha solution $\left(3: 1(\mathrm{v} / \mathrm{v})-\mathrm{H}_{2} \mathrm{SO}_{4} / \mathrm{H}_{2} \mathrm{O}_{2}\right)$ for $\approx 30 \mathrm{~min} @ 70{ }^{\circ} \mathrm{C}$. Then they were flushed with copious amounts of water, sonicated again in MilliQ-water for about $\approx 10 \mathrm{~min}$ and finally stored in MilliQ-water for a maximum of $6 \mathrm{~h}$ until use. Directly before use, they were rinsed again with fresh MilliQ and dry-blown with clean nitrogen (purity 5.0).

\section{Concentration and crystallization scenario for planar, continuously thinning solution films}

If the liquid film consists of a mixture of a volatile solvent and a nonvolatile solute the solvent will evaporate and the solute will continuously get enriched until it remains on the substrate as a final deposit. Because the solvent evaporates with from the film surface the solute primarily gets enriched near the surface. However, this local solute enrichment is counterbalanced by dilution through diffusion away from the surface. The competition between solute enrichment through the loss of solvent via $E$ and solute dilution through diffusive transport quantified through its diffusion constant $D$ can be estimated by a Sherwood number. The Sherwood number for the largest relevant distance, $S h_{t r}$, i.e., on the length scale of a film with the thickness of the transition height $h_{t r}$ is:

$$
S h_{t r}=\left(E \cdot h_{t r}\right) / D=E^{4 / 3} \cdot(2 K)^{-1 / 3} \cdot D^{-1}
$$

If $S h_{t r}<1$, even though the solute enrichment primarily occurs near the film surface, diffusive dilution is very efficient

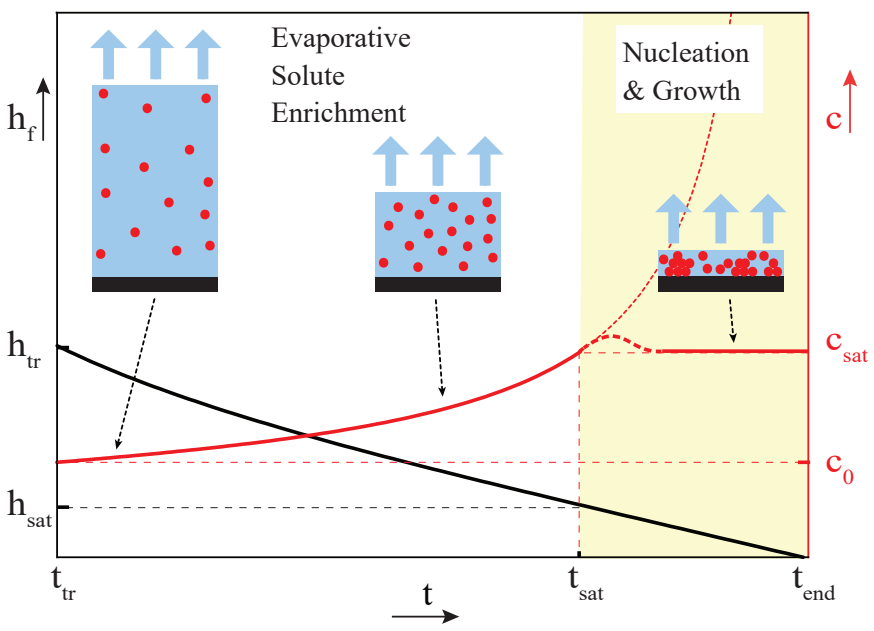

Figure 3: Schematic of the film thinning behaviour and of the concentration evolution in the case of a mixture of a volatile solvent and a non-volatile solute Depicted is the situation for films thinner than the transition height, $h_{t r}$. A homogeneous vertical solute distribution is assumed during solute enrichment as result of the continuous solvent evaporation (i.e., $S h_{t r}<1$, which is the case in most practical cases).

and effectively the vertical solute distribution is rather homogeneous. If $S h_{t r}<1$, i.e. it is small for a film thickness of $h_{t r}$, it is even smaller for thinner films (the distribution within thinner films is even more homogeneous [32]). Typical Sherwood numbers ${ }^{1} S h_{t r}$ for the various solvent/solute systems investigated in this report are significantly smaller than 1 . Therefore, it can be assumed that the vertical distribution of the solute remains rather homogeneous during the entire process of evaporative film thinning respectively solute enrichment. Assuming continuous homogeneous solute enrichment Figure 3 depicts the essential relations between film thinning, solute enrichment, nucleation and growth as it occurs during spin casting of a mixture of a volatile solvent and a nonvolatile solute. It shows the film thickness and the solute concentration as function of the time between the transition time, $t_{t r}$, and complete drying at $t=t_{\text {end }}$. As discussed earlier, for $h_{f}<h_{t r}$ the film thickness decreases due to solvent evaporation approximately linearly with:

$$
h(t) \simeq h_{t r}-E \cdot t
$$

At the same time the solute concentration in the film, $c(h)$, increases with ( $c_{0}$ is the weighing in solute concentration):

$$
c(h) \simeq c_{0} \cdot\left(h_{t r} / h\right)
$$

This means that the solute concentration reaches its saturation value, $c_{\text {sat }}$, at a film thickness $h_{\text {sat }}$ (at a time $t_{\text {sat }}$ ):

$$
h_{s a t} \simeq c_{0} \cdot\left(h_{t r} / c_{s a t}\right)
$$

\footnotetext{
${ }^{1} \mathrm{Sh}_{\text {tr }}\left(\mathrm{C}_{36} \mathrm{H}_{74} /\right.$ toluene $) \leq 0.037, \quad S h_{t r}\left(\mathrm{C}_{30} \mathrm{H}_{60} /\right.$ toluene $) \leq 0.031$, $S h_{t r}\left(\mathrm{C}_{36} \mathrm{H}_{74}\right) / M C H \leq 0.110, \quad S h_{t r}\left(\mathrm{C}_{36} \mathrm{H}_{74} /\right.$ nonane $) \leq 0.008$ and $S h_{t r}\left(\mathrm{NaHCO}_{3} / \mathrm{H}_{2} \mathrm{O}\right) \leq 0.0003$ [44]. Diffusion coefficients $D$ from StokesEinstein-Relation [45]: $D=k_{B} T / 6 \pi \eta R_{0}\left(k_{B}=\right.$ Boltzmann coefficient, $T=$ absolute temperature, $\eta=$ dynamic viscosity, and $R_{0}=$ radius of spherical particle, with $R_{0}=\sqrt{l^{2} / 12}$ for $\mathrm{C}_{30} \mathrm{H}_{62}$ and $\mathrm{C}_{36} \mathrm{H}_{74}$ as cylindrical molecules with lengths $(l)$ of $\sim 4.6 \mathrm{~nm}$ and $\sim 5.6 \mathrm{~nm}$ ).
} 
With a nucleation barrier, right after $t_{\text {sat }}$, the solute concentration will temporarily exceed $c_{\text {sat }}$ until it overcomes the nucleation barrier and the solute starts to crystallize. Then the solute concentration will decrease to $c_{s a t}$ and remain at this value until the system gets completely dry.

\section{Film rupture heights for mixtures of toluene/ $\mathrm{C}_{36} \mathrm{H}_{74}$ and $\mathrm{H}_{2} \mathrm{O} / \mathrm{NaHCO}_{3}$}

Figure 4 presents the hole formation (rupture) behavior of planar films of (a) solutions of $\mathrm{C}_{36} \mathrm{H}_{74}$ in toluene and (b) solutions of $\mathrm{NaHCO}_{3}$ in water. The optical imaging shows that after liquid deposition on the rotating substrate, in both cases and for all weighing in solute concentrations, $c_{0}$, all solutions first form a whole, homogeneously planar film. During evaporative thinning all liquid films remain whole as long as they are thicker than about $50 \mathrm{~nm}$. With thinner films the wetting behavior depends on the solute concentration. Pure solvent films remain whole during the entire film thinning until all the liquid has evaporated. Films of solutions form holes and rupture as soon as they become thinner than a specific film thickness, $h_{\text {rupture }}$. This $h_{\text {rupture }}$ depends on the solution composition, in particular on the solute concentration. If the weighing in solute concentration, $c_{0}$, is very low, $h_{\text {rupture }}$ is rather small. $h_{\text {rupture }}$ increases linearly with $c_{0}$ until it reaches a maximum rupture thickness of $h_{\text {rupture }} \approx 50 \mathrm{~nm}$. Upon increasing $c_{0}$ further, $h_{\text {rupture }}$ remains constant at $\approx 50 \mathrm{~nm}$.

The optical investigations during and after the film thinning as well as AFM studies on the dry samples show that the hole formation starts at spots, where the solute crystallizes at the interface between substrate and liquid film. $\mathrm{C}_{36} \mathrm{H}_{74}$ forms the typical dendritic 2-dimensional mono- or multilayer aggregates that are described already in the literature elsewhere [4649]. $\mathrm{NaHCO}_{3}$ forms small crystals with the typical monoclinic structure [50]. The crystallization of anorganic salts from evaporating aqueous solutions is described elsewhere [51, 52].

\section{Film rupture thicknesses for various different binary mixtures}

Figure 5(a) presents the hole formation behavior i.e., the film rupture thicknesses, $h_{\text {rupture }}$, of five different solutions as a function of the solute concentration. All pure solvents wet the substrate completely. As already presented for two solutions in Figure 4 , in all cases $h_{\text {rupture }}$ increases linearly with the solute concentration, $c_{0}$. As soon as $h_{\text {rupture }}$ reaches $h_{\text {rupture }}^{\text {max }} \approx 50 \mathrm{~nm}$, it remains constant, even as $c_{0}$ is increased further. The solubilities of the solutes in the different solvents vary considerably. Accordingly, the transition from the increasing $h_{\text {rupture }}$ to the plateau with $h_{\text {rupture }}^{\text {max }} \approx 50 \mathrm{~nm}$ is orders of magnitude different for different solutions. Figure 5(b) shows the same data in a plot of $h_{\text {rupture }}$ vs. $h_{\text {sat }}$. The dashed line shows $h_{\text {rupture }}=h_{\text {sat }}$. With this rescaling all data collapse into one universal behaviour. $h_{\text {rupture }}$ increases linearly with $h_{\text {sat }}$ up to a maximum value of $h_{\text {rupture }}^{\max }$ and then remains at this value, even if $h_{\text {sat }}$ increases (a)

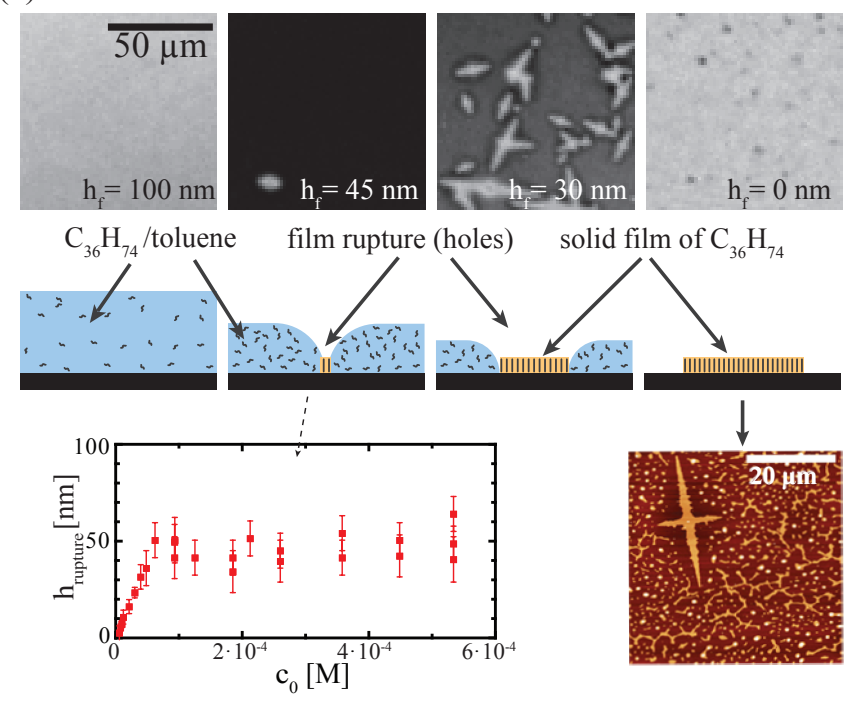

(b)

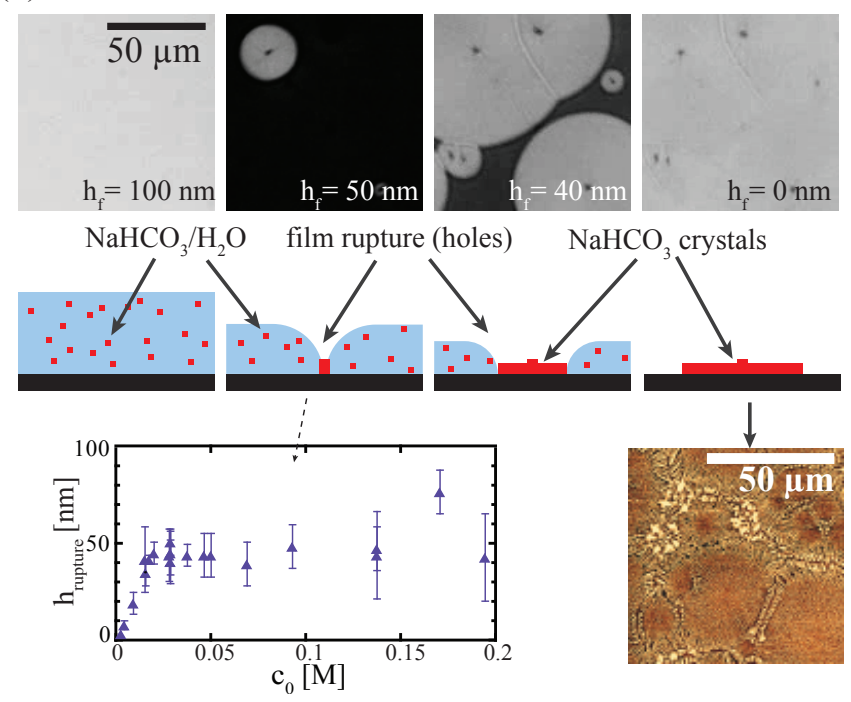

Figure 4: Rupture behavior of films of (a) mixtures of $\mathrm{C}_{36} \mathrm{H}_{74}$ /toluene $\left(c_{0}=\right.$ $\left.6 \cdot 10^{-4} \mathrm{M}\right)$ and of (b) $\mathrm{NaHCO}_{3} / \mathrm{H}_{2} \mathrm{O}\left(c_{0}=0.09 \mathrm{M}\right)$. The top rows show examples of images recorded as the films become thinner due to solvent evaporation. The cartoons depict the corresponding cross sections through the films indicating the crystallization of the solute at the substrate/film interface and the film rupture (hole formation) at this location. The plots show $h_{\text {rupture }}$, the film heights at which hole formation is observed/commences, as function of $c_{0}$, the weighing in concentration of the solute. Also shown are AFM images of the dry solute on the substrates after complete solvent evaporation. Each data point represents the results of several experiments (errors estimated from rupture statistics and thickness resolution). Different data points at the same $c_{0}$ represent results from different measurement series.

further. Optical imaging reveals that the holes in the film always develop where solute crystals start to grow at the substrate/liquid interface for the the case when $h_{\text {sat }}<h_{\text {rupture }}^{\text {max }}$. For $h_{\text {sat }}<h_{\text {rupture }}^{\text {max }}$ solute crystals probably grow already as soon $c>c_{\text {sat }}$ for films thicker than $h_{\text {rupture }}^{\max }$. In this case hole formation commences as soon as $h_{\text {sat }} \leq h_{\text {rupture }}^{\text {max }}$ at locations with solute crystals at the interface between film and substrate. These scenarios are depicted in the cartoons of Figure 5(b). 
(a)

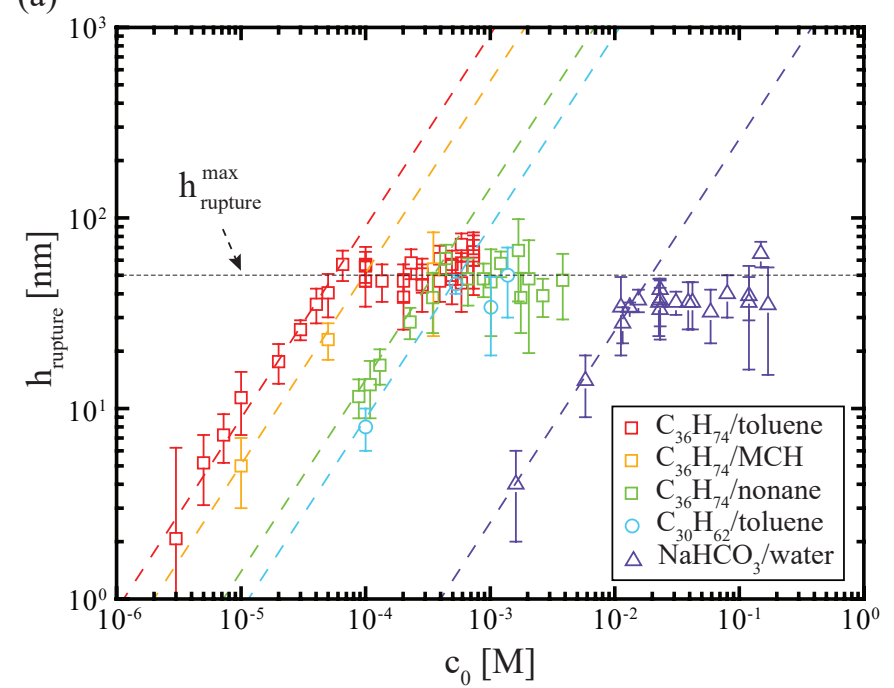

(b)

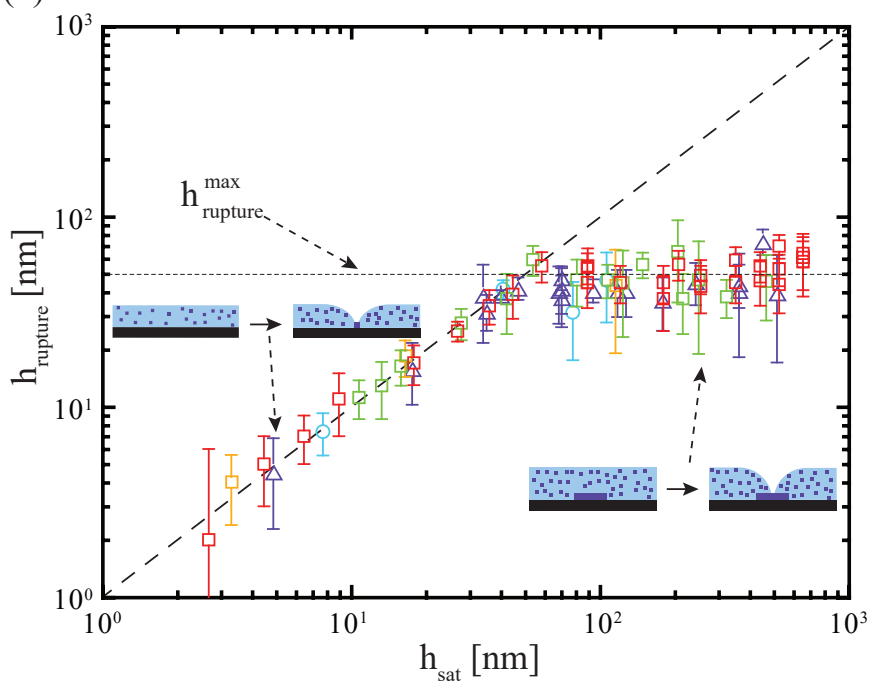

Figure 5: (a) Film rupture (hole formation) thicknesses, $h_{\text {rupture }}$, as function of the weighing in solute concentrations, $c_{0}$, for various solutions: $\mathrm{C}_{36} \mathrm{H}_{74} /$ toluene $\left(c_{\text {sat }}=1.8 \cdot 10^{-3} \mathrm{M}, h_{\text {tr }} \approx 5 \mu \mathrm{m}\right), \mathrm{C}_{36} \mathrm{H}_{74} / M C H\left(c_{\text {sat }}=\right.$ $\left.1.4 \cdot 10^{-2} \mathrm{M}, h_{t r} \approx 7 \mu \mathrm{m}\right)(\mathrm{MCH}-$ methylcyclohexane $), \mathrm{C}_{36} \mathrm{H}_{74} /$ nonane $\left(c_{\text {sat }}=2.5 \cdot 10^{-2} \mathrm{M}, h_{t r} \approx 3 \mu \mathrm{m}\right), \mathrm{C}_{30} \mathrm{H}_{62} /$ toluene $\left(c_{\text {sat }}=5.5 \cdot 10^{-2} \mathrm{M}, h_{t r} \approx\right.$ $5 \mu \mathrm{m}), \mathrm{NaHCO}_{3} /$ water $\left(c_{\text {sat }}=1.1 \mathrm{M}, h_{t r} \approx 3 \mu \mathrm{m}\right)$. The dashed lines show $h_{\text {sat }}$, the film thicknesses, where the solute concentration reaches its saturation. (b) Plot of $h_{\text {rupture }}$ as function of $h_{\text {sat }}$. The dashed line shows $h_{\text {rupture }}=h_{\text {sat }}$. The cartoons show how for $h_{\text {sat }}<h_{\text {rupture }}^{\text {max }}$ solute crystallization and hole formation occurs approximately simultaneously, whereas for $h_{\text {sat }}>h_{\text {rupture }}^{\max }$ solute precipitation occurs before film rupture.

\section{Discussion}

Figure 5(a) shows that for small solute weighing in concentrations, $c_{0}$, the rupture film thicknesses, $h_{\text {rupture }}$, increase proportional to $c_{0}$. As soon as $h_{\text {rupture }} \approx 50 \mathrm{~nm}$, it remains constant at this value $h_{\text {rupture }}^{\max }$ (independent of $c_{0}$ ). The linear increase of $h_{\text {rupture }}$ with $c_{0}$ and the limit with $h_{\text {rupture }}^{\text {max }} \approx 50 \mathrm{~nm}$ is observed for all the various combinations of solutes and solvents investigated although the transition between the linear increase and the plateau behavior occurs with $c_{0}$, which are different by orders of magnitude.
The key to understanding this behaviour is the solute enrichment during the evaporative film thinning in a spin cast configuration, which has recently been analyzed quantitatively[32, 33]. Figure 3 shows qualitatively the increase of the solute concentration as the film becomes thinner due to the solvent evaporation. With the setup shown in Figure 1 the evaporation rates, $E$, of the various solutions can be measured (Equation $3)$. Thus, the increase of the solute concentration can be determined (Equation 4) and $h_{\text {sat }}$ can be calculated. The measurements show that the transition between the range with a linear increase of $h_{\text {rupture }} \propto c_{0}$ and the plateau behaviour with $h_{\text {rupture }}=$ const $=h_{\text {rupture }}^{\text {max }}$ occurs in all cases when the solute concentration within the film reaches its saturation value, $c_{\text {sat }}$, at film thickness of $h_{f} \approx 50 \mathrm{~nm}$. Taking this into account the rupturing behaviors of all the different solutions can be rescaled and plotted universally as depicted in Figure 5(a).

The time-resolved optical imaging of the films during the film thinning and rupturing reveals the possible reason for the hole formation. It appears as if film rupturing always occurs at locations, where the solute is forming (or has already formed) crystals at the interface between substrate and liquid film. This indicates the following film rupture scenario: As soon as the solute becomes sufficiently supersaturated (i.e., $c=c_{\text {sat }}+\delta c$, where $\delta c$ accounts for the extra concentration to overcome the nucleation barrier) it starts to crystallize. Basically, nucleation and crystal growth might occur at three different locations: 1.) at the substrate surface, 2.) within the solution, or 3.) at the film surface. During nucleation and early crystal growth the crystals are very small and they are not directly visible with optical microscopy. As soon as they grow to micron size they may become optically visible and their location at this stage indicates where their nucleation and early growth may have occurred.

Alkanes form quasi-2-D mono- or multilayer domains, which indeed can be imaged by optical microscopy [46, 47]. For the alkane solutions investigated for this report, with initial alkane concentrations substantially lower than the saturation concentration $\left(c_{0}<0.1 c_{\text {sat }}\right)$, we do not observe any alkane domains before the film ruptures (neither within the solution nor at the substrate/film or the film/air interface). On the other hand, we find that the film always ruptures and forms a hole at a spot, where an alkane domain is attached to the substrate. This domain can be seen either already during the widening of the hole or later on the dry substrate. This is the scenario depicted in Fig. 4(a). It is corroborated by experiments with films of very high initial alkane concentrations $\left(c_{0}>0.1 c_{s a t}\right)$, which are not the subject matter of this report, because their thinning behaviour is fundamentally different to the case described in this report ${ }^{2}$. Nevertheless, films with very high initial alkane concentrations $\left(0.1 c_{\text {sat }}<c_{0}<0.9 c_{\text {sat }}\right)$ show the following relevant behaviour:

1.) Occasionally alkane multi-layer domains are observed, which are attached to the substrate and underneath (i.e., covered by) a rather thick ( $>100 \mathrm{~nm})$, whole (unruptured)

\footnotetext{
${ }^{2}$ With high initial alkane concentrations the liquid film surface becomes ondulated, supposedly because of Marangoni flows. These observations are well beyond the scope of this manuscript (the rupture of the films) and will be the topic of another report.
} 
alkane/solvent film. As soon as the film on top of the multilayer domains becomes sufficiently thin $(\approx 50 \mathrm{~nm})$, it ruptures at the location of these domains.

2.) Repeatedly alkane domains are observed, which are attached to the film/air interface of a rather thick $(>>100 \mathrm{~nm})$ alkane/solvent film. The location of these domains can be identified, because a) they are moving laterally relative to the substrate surface (floating) and b) they visibly distort the film surface in their vicinity. Again, the film ruptures preferentially at the location of these floating domain as soon as it is thin enough $(\approx 50 \mathrm{~nm})$.

3.) Very rarely alkane domains are observed, which float within a thick (>> $100 \mathrm{~nm}$ ) film. These domains move laterally relative to the substrate surface without causing a film surface distortion. This case is so rare that we do not have conclusive experimental data on how these domains influence film rupture. It appears as if these floating domains attach to one of the interfaces during film thinning and eventually the film ruptures as described in the two cases with the domains attached to the interfaces.

As in the case of the alkane solutions, with solutions of $\mathrm{NaHCO}_{3} /$ water we also never see crystals before film rupture. And, as in the case of the alkanes, the film always ruptures and forms a hole at a spot, where we always find (later) optically (or by AFM) a crystal of $\mathrm{NaHCO}_{3}$ (see for example Fig. 4(b)). This crystal is usually larger than the other crystals in its vicinity (which result from the receding three phase contact line). With $\mathrm{NaHCO}_{3}$ also the three nucleation and growth scenarios at the three different locations are conceivable, as discussed above for the alkanes. However, with $\mathrm{NaHCO}_{3}$ these cases cannot be distinguished by optical microscopy in a way as described for the alkanes, because $\mathrm{NaHCO}_{3}$ forms 3-D crystals. Therefore, as soon as the crystals are large enough to be optically observable, they already are larger (higher) than the thickness of the film in which they grow.

It should be mentioned that the results of a few selective experiments with aqueous solutions of $\mathrm{NaCl}$ agree with the findings obtained with $\mathrm{NaHCO}_{3}$ and with the alkanes. By and large, the observation with the alkane domains agrees with the expectation that the crystallization preferentially commences at the film/substrate interface, because heterogeneous nucleation at this interface is more favorable than homogeneous nucleation within the film or heterogeneous nucleation at the film/air interface [53].

The observations indicate that, if the film is sufficiently thin (a few tens of nm), film rupture is initiated at a spot, where the interface between film and substrate is locally modified by a crystal. The local growth of a crystal effectively changes a) the local wetting properties and b) it reduces locally the film thickness. In the case of long chain alkanes, the local crystal is a flat mono- or multilayer domain, typically only a few $\mathrm{nm}$ thick. Therefore the local film is not much thinner (on the scale of $h_{\text {rupture }}^{\text {max }}$ ) than the film on the substrate without the alkane domain and the locally different wetting properties might be more important than the locally thinner film. It is known that surfaces of solid long chain alkanes are not wetted by liquid short chain alkanes, such as nonane ([46, 54-56]). It can be assumed that they are also not wetted completely by other hydrophobic liquids, such as toluene or methylcyclohexane. Very little is known about the wettability of anorganic crystals $\left(\mathrm{NaHCO}_{3}\right)$ by anorganic solute/solvent mixtures $\left(\mathrm{NaHCO}_{3} /\right.$ water $)$, with the solute and the crystals being identical substances. The observed rapidly retreating contact line of the $\mathrm{NaHCO}_{3} /$ water solutions as soon as the film is thinner than $h_{\text {rupture }}^{\text {max }}$ and has ruptured indicates that the $\mathrm{NaHCO}_{3} /$ water solutions do not wet completely substrate surfaces that are (partially) covered by $\mathrm{NaHCO}_{3}$ crystals. In any case, with a globally continuously thinning film, the local non-wetting properties in combination with the locally thinner film eventually will lead to film rupture either by a spinodal $[20,57]$ or a nucleated process $[24,58]$.

The wetting behavior of films is affected by VdW interactions and in the non-wetting case film rupture driven by $\mathrm{VdW}$ interactiosn typically occurs for film thicknesses of a few tens of nanometers. This is what we observe $\left(h_{\text {rupture }}^{\max }\right)$. This is also in agreement with the cases of low solute concentrations, when the films rupture instantaneously as soon as the substrate (locally) switches from wetting to non-wetting due to the local growth of a crystal at the interface between film and substrate $\left(h_{f}<h_{\text {rupture }}^{\max }\right.$ for low $\left.c_{0}\right)$.

Based on the assumption that $\mathrm{VdW}$ interactions drive the film rupture we performed experiments with different Hamaker constants. We used substrates with oxide layers of different thicknesses (natural oxide layers of only $\approx 1.5 \mathrm{~nm}$ thickness, artificial oxide layers $50 \mathrm{~nm}$ and $100 \mathrm{~nm}$ thick). These variations are part of the data presented in Figures 4 and 5. They are not indicated individually, because there is no systematic correlation between the variations and the observed behaviour. Aside from modifications of the substrate surface, the VdW-interactions of systems with water as solvent should be different to those with toluene (the refractive indices and thus the Hamaker constants of both systems are significantly different). Figure 5 shows that within the errors the results are not sensitive to these variations. The results with different substrates and solvents do neither support nor exclude VdW-interactions as main cause for the film rupture, because the errors for $h_{\text {rupture }}^{\text {max }}$ are quite large. Even if differences in the VdW-interactions had an impact on $h_{\text {rupture }}^{\text {max }}$, this hardly could be derived from the data.

\section{Summary and Conclusion}

A setup has been designed and implemented to optically image time-resolved the thinning and rupture behaviour of thin planar films of binary mixtures of a volatile solvent and a nonvolatile solute on planar substrates in a spin cast configuration. The solutes form crystals as soon as the concentration exceeds a know saturation value. The focus is on the wetting/rupturing behavior of very thin films. The thinning behavior and timedependent variation of the film composition in the applied spin cast configuration has been studied and is well-known. At the very late stages of the spin cast process, hydrodynamic flow can be neglected. The system is essentially a planar liquid film, which is thinning due to a constant evaporation rate. It is observed that depending on the type of solvent/solute mixture and solute concentration the films rupture at certain film 
thicknesses. This behaviour is analyzed and discussed in some detail.

In conclusion, the experimental results and their analysis reveal three significant, important, and hitherto unknown findings: 1.) the films rupture if a.) the solute concentration reaches its saturation value and if b.) the film is as thick or thinner than a certain film thickness, $h_{\text {rupture }}^{\max }$, 2.) The rupture behaviour is remarkably universal in view of the really very large variation of initial solute concentrations and different combinations of solute/solvent, 3.) In all cases $h_{\text {rupture }}^{\max }$ has a value somewhere between more than $10 \mathrm{~nm}$ and less than $100 \mathrm{~nm}$. Supposedly the crystallization of the solute at the film/substrate interface alters locally the wetting properties. This leads to the film rupture at this location. The value of $h_{\text {rupture }}^{\text {max }}$ is an indication and consistent with $\mathrm{VdW}$-interactions as main reason for the film rupture. It can be assumed that such a film rupture behavior occurs very often. For instance, most natural aqueous solutions contain non-volatile, precipitating/crystallizing components such as salts. Regarding application, fhe findings can also be used to tailor-make solutions to trigger the rupture of evaporating films at specified thicknesses with specific hole formation scenarios (e.g. by adjusting how the solute crystallization/precipitation occurs).

\section{Acknowledgements}

For assistance with the AFM measurements we thank José Danglad-Flores, and acknowledge helpful discussions with Stefan Karpitschka. We also thank Reinhard Lipowsky for general support.

\section{References}

[1] P.-G. De Gennes, Wetting: statics and dynamics, Reviews of modern physics 57 (3) (1985) 827

[2] R. Seemann, S. Herminghaus, K. Jacobs, Dewetting patterns and molecular forces: A reconciliation, Physical Review Letters 86 (24) (2001) 5534.

[3] U. Thiele, Open questions and promising new fields in dewetting, The European Physical Journal E 12 (3) (2003) 409-416.

[4] D. Bonn, J. Eggers, J. Indekeu, J. Meunier, E. Rolley, Wetting and spreading, Reviews of modern physics 81 (2) (2009) 739.

[5] J. H. Snoeijer, B. Andreotti, Moving contact lines: scales, regimes, and dynamical transitions, Annual review of fluid mechanics 45 (2013) 269292, doi:10.1146/annurev-fluid-011212-140734.

[6] E. Schäffer, T. Thurn-Albrecht, T. P. Russell, U. Steiner, Electrically induced structure formation and pattern transfer, Nature 403 (6772) (2000) 874.

[7] K. Kargupta, A. Sharma, Templating of thin films induced by dewetting on patterned surfaces, Physical Review Letters 86 (20) (2001) 4536.

[8] A. Sharma, Many paths to dewetting of thin films: anatomy and physiology of surface instability, The European Physical Journal E 12 (3) (2003) 397-408.

[9] R. P. Wool, Self-healing materials: a review, Soft Matter 4 (3) (2008) 400-418.

[10] T. D. Lee, A. U. Ebong, A review of thin film solar cell technologies and challenges, Renewable and Sustainable Energy Reviews 70 (2017) 12861297.

[11] A. M. Telford, S. C. Thickett, C. Neto, Functional patterned coatings by thin polymer film dewetting, Journal of colloid and interface science 507 (2017) 453-469.

[12] A. Sheludko, Thin liquid films, Advances in Colloid and Interface Science 1 (4) (1967) 391-464.
[13] M. B. Williams, S. H. Davis, Nonlinear theory of film rupture, Journal of Colloid and Interface Science 90 (1) (1982) 220-228.

[14] A. Oron, S. H. Davis, S. G. Bankoff, Long-scale evolution of thin liquid films, Reviews of modern physics 69 (3) (1997) 931.

[15] G. Reiter, A. Sharma, A. Casoli, M.-O. David, R. Khanna, P. Auroy, Thin film instability induced by long-range forces, Langmuir 15 (7) (1999) 2551-2558.

[16] L. W. Schwartz, R. V. Roy, R. R. Eley, S. Petrash, Dewetting patterns in a drying liquid film, Journal of colloid and interface science 234 (2) (2001) 363-374.

[17] N. Mulji, S. Chandra, Rupture and dewetting of water films on solid surfaces, Journal of colloid and interface science 352 (1) (2010) 194-201.

[18] W. Li, D. Lan, Y. Wang, Dewetting-mediated pattern formation inside the coffee ring, Physical Review E 95 (4) (2017) 042607.

[19] E. D. Manev, A. V. Nguyen, Critical thickness of microscopic thin liquid films, Advances in colloid and interface science 114 (2005) 133-146.

[20] U. Thiele, M. Mertig, W. Pompe, Dewetting of an evaporating thin liquid film: Heterogeneous nucleation and surface instability, Physical review letters 80 (13) (1998) 2869.

[21] G. Reiter, Dewetting of thin polymer films, Physical Review Letters 68 (1) (1992) 75 .

[22] G. Reiter, Unstable thin polymer films: rupture and dewetting processes, Langmuir 9 (5) (1993) 1344-1351.

[23] G. Reiter, P. Auroy, L. Auvray, Instabilities of thin polymer films on layers of chemically identical grafted molecules, Macromolecules 29 (6) (1996) 2150-2157.

[24] J. Bischof, D. Scherer, S. Herminghaus, P. Leiderer, Dewetting modes of thin metallic films: nucleation of holes and spinodal dewetting, Physical Review Letters 77 (8) (1996) 1536.

[25] R. Seemann, S. Herminghaus, K. Jacobs, Gaining control of pattern formation of dewetting liquid films, Journal of Physics: Condensed Matter 13 (21) (2001) 4925.

[26] X. Gu, D. Raghavan, J. F. Douglas, A. Karim, Hole-growth instability in the dewetting of evaporating polymer solution films, Journal of Polymer Science Part B: Polymer Physics 40 (24) (2002) 2825-2832.

[27] L. Fisher, A. Golovin, Nonlinear stability analysis of a two-layer thin liquid film: Dewetting and autophobic behavior, Journal of colloid and interface science 291 (2) (2005) 515-528.

[28] U. Thiele, Thin film evolution equations from (evaporating) dewetting liquid layers to epitaxial growth, Journal of Physics: Condensed Matter 22 (8) (2010) 084019.

[29] D. Peschka, A. Münch, B. Niethammer, Thin-film rupture for large slip, Journal of Engineering Mathematics 66 (1-3) (2010) 33-51.

[30] H. Ji, T. P. Witelski, Finite-time thin film rupture driven by modified evaporative loss, Physica D: Nonlinear Phenomena 342 (2017) 1-15.

[31] U. Thiele, D. V. Todorova, H. Lopez, Gradient dynamics description for films of mixtures and suspensions: dewetting triggered by coupled film height and concentration fluctuations, Physical review letters 111 (11) (2013) 117801.

[32] S. Karpitschka, C. M. Weber, H. Riegler, Spin casting of dilute solutions: Vertical composition profile during hydrodynamic-evaporative film thinning, Chemical Engineering Science 129 (2015) 243-248, doi: 10.1016/j.ces.2015.01.028.

[33] J. Danglad-Flores, S. Eickelmann, H. Riegler, Deposition of polymer films by spin casting: A quantitative analysis, Chemical Engineering Science 179 (2018) 257-264, doi:10.1016/j.ces.2018.01.012.

[34] S. Ebbens, R. Hodgkinson, A. J. Parnell, A. Dunbar, S. J. Martin, P. D. Topham, N. Clarke, J. R. Howse, In situ imaging and height reconstruction of phase separation processes in polymer blends during spin coating, ACS nano 5 (6) (2011) 5124-5131.

[35] D. T. Toolan, A. Dunbar, S. Ebbens, N. Clarke, P. D. Topham, J. R. Howse, et al., Direct observation of morphological development during the spin-coating of polystyrene-poly (methyl methacrylate) polymer blends, Journal of Polymer Science Part B: Polymer Physics 51 (11) (2013) 875-881.

[36] D. T. Toolan, A. J. Parnell, P. D. Topham, J. R. Howse, Directed phase separation of PFO: PS blends during spin-coating using feedback controlled in situ stroboscopic fluorescence microscopy, Journal of Materials Chemistry A 1 (11) (2013) 3587-3592.

[37] D. T. Toolan, J. R. Howse, Development of in situ studies of spin coated polymer films, Journal of Materials Chemistry C 1 (4) (2013) 603-616. 
[38] S. Eickelmann, Real-Time Imaging Study of Ultra-Thin Liquid Films in a Spin-Cast Configuration, Master's thesis, University of Potsdam, Potsdam, Germany, 2014.

[39] A. G. Emslie, F. T. Bonner, L. G. Peck, Flow of a viscous liquid on a rotating disk, Journal of Applied Physics 29 (5) (1958) 858-862.

[40] D. Meyerhofer, Characteristics of resist films produced by spinning, Journal of Applied Physics 49 (7) (1978) 3993-3997.

[41] R. Köhler, P. Lazar, H. Riegler, Optical imaging of thin films with molecular depth resolution, Applied physics letters 89 (24) (2006) 241906.

[42] S. Karpitschka, E. Dietrich, J. R. T. Seddon, H. J. W. Zandvliet, D. Lohse, H. Riegler, Nonintrusive Optical Visualization of Surface Nanobubbles, Phys. Rev. Lett 109 (2012) 066102.

[43] F. Horowitz, E. Yeatman, E. Dawnay, A. Fardad, Real-time optical monitoring of spin coating, Journal de Physique III 3 (11) (1993) 2059-2063.

[44] R. C. Weast, M. J. Astle, W. H. Beyer, et al., CRC handbook of chemistry and physics, vol. 1990, CRC press, Boca raton FL, 1989.

[45] A. Einstein, Über die von der molekularkinetischen Theorie der Wärme geforderte Bewegung von in ruhenden Flüssigkeiten suspendierten Teilchen, Annalen der physik 322 (8) (1905) 549-560.

[46] A. Holzwarth, S. Leporatti, H. Riegler, Molecular ordering and domain morphology of molecularly thin triacontane films at $\mathrm{SiO} 2$ /air interfaces, EPL Europhysics Letters 52 (6) (2000) 653.

[47] S. N. Magonov, N. A. Yerina, Visualization of nanostructures with atomic force microscopy, in: Handbook of microscopy for nanotechnology, Springer, 113-155, 2005.

[48] L. Pithan, E. Meister, C. Jin, C. Weber, A. Zykov, K. Sauer, W. Brütting, H. Riegler, A. Opitz, S. Kowarik, Thermally driven smoothening of molecular thin films: Structural transitions in $\mathrm{n}$-alkane layers studied in real-time, The Journal of Chemical Physics 143 (16) (2015) 164707.

[49] T. P. Corrales, M. Bai, V. del Campo, P. Homm, P. Ferrari, A. Diama, C. Wagner, H. Taub, K. Knorr, M. Deutsch, M. J. Retamal, U. G. Volkmann, P. Huber, Spontaneous Formation of Nanopatterns in VelocityDependent Dip-Coated Organic Films: From Dragonflies to Stripes, ACS Nano 8 (10) (2014) 9954-9963, doi:10.1021/nn5014534.

[50] W. Zachariasen, The crystal lattice of sodium bicarbonate, $\mathrm{NaHCO} 3$, The Journal of Chemical Physics 1 (9) (1933) 634-639.

[51] N. Shahidzadeh-Bonn, S. Rafa, D. Bonn, G. Wegdam, Salt Crystallization during Evaporation: Impact of Interfacial Properties, Langmuir 24 (16) (2008) 8599-8605, doi:10.1021/1a8005629.

[52] F. Lequien, V. Souli, G. Moine, A. Lequien, D. Feron, P. Prene, H. Moehwald, H. Riegler, T. Zemb, Corrosion influence on the evaporation of sessile droplet, Colloids and Surfaces A: Physicochemical and Engineering Aspects 546 (2018) 59 - 66, ISSN 0927-7757, doi: https://doi.org/10.1016/j.colsurfa.2018.02.047.

[53] R. Perez-Garcia, H. Riegler, Controlled Self-Organized Positioning of Small Aggregates by Patterns of (Sub) nanosized Active Sites, Crystal Growth \& Design 17 (4) (2017) 1870-1875.

[54] H. Schollmeyer, B. Struth, H. Riegler, Long chain n-alkanes at SiO2/air interfaces: Molecular ordering, annealing, and surface freezing of triacontane in the case of excess and submonolayer coverage, Langmuir 19 (12) (2003) 5042-5051.

[55] H. Schollmeyer, B. Ocko, H. Riegler, Surface freezing of triacontane at $\mathrm{SiO}$ x/air interfaces: Submonolayer coverage, Langmuir 18 (11) (2002) 4351-4355.

[56] H. Riegler, R. Köhler, How pre-melting on surrounding interfaces broadens solid-liquid phase transitions, Nature physics 3 (12) (2007) 890.

[57] R. Xie, A. Karim, J. F. Douglas, C. C. Han, R. A. Weiss, Spinodal dewetting of thin polymer films, Physical Review Letters 81 (6) (1998) 1251.

[58] U. Thiele, M. G. Velarde, K. Neuffer, Dewetting: Film rupture by nucleation in the spinodal regime, Physical review letters 87 (1) (2001) 016104. 